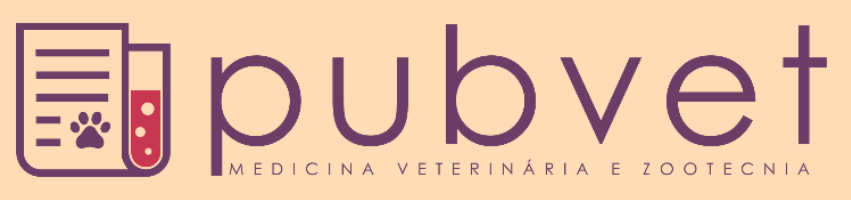

https://doi.org/10.31533/pubvet.v13n7a380.1-5

\title{
Esofagotomia torácica para remoção de corpo estranho em um cão: Relato de caso
}

\author{
Francisco de Assis Araújo Camelo Júnior ${ }^{1} \bullet$, Thiago Franco ${ }^{2}$, Virginia Machado Feijó ${ }^{2} \bullet$, \\ Risciela Salardi Alves de Brito ${ }^{\circ}$, Humberto Zanusso de Medeiros ${ }^{4}$, Maria Gabriela de Mello \\ Fonseca $^{4}{ }^{\circ}$, Paula Andreuchetti Madruga ${ }^{\circ}$, Dilene Formentin Priario ${ }^{\circ}{ }^{\circ}$, Sérgio Jorge ${ }^{6}$, $^{\circ}$ \\ Jorge Squeff Filho $^{70}$
}

${ }^{I}$ Médico Veterinário, autônomo, Universidade Federal de Pelotas (UFPel), Pelotas, Rio Grande do Sul, Brasil.

${ }^{2}$ Discente em Medicina Veterinária, Universidade Federal de Pelotas (UFPel), Pelotas, Rio Grande do Sul, Brasil

${ }^{3}$ Médica Veterinária, doutoranda no Programa de Pós-graduação da Medicina Veterinária da UFPel, Pelotas, Rio Grande do Sul, Brasil.

${ }^{4}$ Médico Veterinário, autônomo, UFPel, Rio Grande do Sul, Brasil.

${ }^{5}$ Médica Veterinária, autônoma, Universidade Federal do Pampa, Rio Grande do Sul, Brasil.

${ }^{6}$ Médico Veterinário e Biólogo, Pós-doutorando no Programa de Pós-graduação da Biotecnologia da UFPel, Rio Grande do Sul, Brasil.

${ }^{7}$ Médico Veterinário, mestre em ciência animal da UFPel, Pelotas, Rio Grande do Sul, Brasil.

*Autor para correspondência, E-mail: junior_camelo01@hotmail.com

\begin{abstract}
Resumo. Os corpos estranhos são objetos que podem ocasionar obstrução ou desconforto ao animal por geralmente serem grandes e apresentarem pontas finas e afiadas que podem perfurar órgãos, como a mucosa esofágica. Os cães são mais acometidos que os felinos devido aos hábitos alimentares indiscriminados. O esôfago apresenta uma anatomia tubular relativamente estreita, responsável por conduzir alimentos da faringe ao estômago. Os sinais clínicos de obstrução variam e dependem da duração, localização, grau de obstrução e acometimento da parede esofágica. O diagnóstico baseia-se no histórico clínico do paciente, radiografia ou endoscopia. A remoção cirúrgica é amplamente utilizada pela gama de possibilidades e a escolha do procedimento e protocolo varia de acordo com a localização, tamanho/forma do corpo estranho, e grau da obstrução, como a toracotomia. O objetivo do presente trabalho é relatar um caso em que a esofagotomia torácica foi optada para a remoção de um corpo estranho que obstruía o lúmen esofágico na porção torácica de um cão.
\end{abstract}

Palavras chave: cirurgia, esôfago, obstrução, tórax

\section{Esophagotomy thoracic body removal strange in a dog: Case report}

\begin{abstract}
Foreign bodies are objects that can cause obstruction or discomfort to the animal and, in general, have sharp, sharp tips that can pierce an esophageal mucosa. Dogs are more affected than cats because of indiscriminate foods. The esophagus presents a relatively tubular anatomy, responsible for feeding the pharynx to the stomach. The clinical signs of obstruction vary and depend on the duration, location, degree of obstruction and involvement of the esophageal wall. The diagnosis is based on the patient's clinical history, radiography or endoscopy. Surgical removal is widely used for the range of possibilities, whose procedure of choice and protocol varies according to the location, size / shape of the foreign body and degree of obstruction, such as thoracotomy. The case of this work is to report a case of a thoracic esophagotomy that may be useful for the removal of a foreign body that may obstruct the thoracic functions of a dog.
\end{abstract}

Keywords: surgery, esophagus, obstruction, thorax 


\section{Esofagotomía torácico para remoción de cuerpo extraño em un perro: Reporte de caso}

Resumen. Los cuerpos extraños son objetos que pueden ocasionar obstrucción o incomodidad al animal por ser generalmente grandes y presentar puntas finas y afiladas que pueden perforar la mucosa esofágica. Los perros son más acometidos que los felinos debido a los hábitos alimentarios indiscriminados. El esófago presenta una anatomía tubular relativamente estrecha, responsable de conducir alimentos de la faringe al estómago. Los signos clínicos de obstrucción varían y dependen de la duración, ubicación, grado de obstrucción y afectación de la pared esofágica. El diagnóstico se basa en el historial clínico del paciente, la radiografía o la endoscopia. La remoción quirúrgica es ampliamente utilizada por la gama de posibilidades, cuya elección del procedimiento y protocolo varía de acuerdo con la ubicación, tamaño/forma del cuerpo extraño, y grado de la obstrucción, como toracotomía. El objetivo del presente trabajo es relatar un caso en que la esofagotomía torácica fue optada para la remoción de un cuerpo extraño que obstruía el lumen esofágico en la porción torácica de un perro.

Palabras clave: cirugía, esófago, obstrucción, tórax

\section{Introdução}

Os corpos estranhos esofágicos são objetos que podem ocasionar obstrução ou desconforto ao animal por geralmente serem grandes e apresentarem pontas finas e afiadas que podem perfurar a mucosa esofágica, onde comumente são observados grandes pedaços de ossos ou objeto metálico afiado (Tans \& Rawlings, 2011). Os cães, são mais acometidos que os felinos, devido aos hábitos alimentares, jovens e de pequeno porte apresentam uma incidência maior (Nelson \& Couto, 2015). Todavia, deve-se considerar o histórico e sinais clínicos (Tans \& Rawlings, 2011).

O esôfago apresenta uma anatomia tubular relativamente estreita, responsável por conduzir alimentos da faringe ao estômago, composto pelas porções cervical, torácica e abdominal (Fossum, 2014). O trecho cranial esofágico, na base do coração e na região epifrenia são os que apresentam maior acometimento devido às estruturas extra-esofágicas se limitarem a sua dilatação (Juvet et al., 2010).

Os sinais clínicos variam e dependem da duração, localização, grau de obstrução e acometimento da parede esofágica (Fossum, 2014). Os sinais mais comuns são salivação excessiva, reflexos espasmódicos, mímica de vômito, regurgitação após a alimentação, inquietação, dor e angústia respiratória (Rodríguez et al., 2012). Na maioria dos casos as obstruções completas ocasionam regurgitação de sólidos e líquidos, ao passo que as obstruções parciais podem permitir a retenção de líquidos. Pode ainda ocorrer dispneia aguda em casos onde o corpo estranho esteja alojado e comprimindo vias aéreas ou quando progrediu para uma pneumonia por aspiração (Tans, 2005). Em casos de perfuração, o animal pode apresentar febre, depressão e/ou anorexia (Tans, 2005).

O diagnóstico baseia-se no histórico clínico fornecido pelo tutor do paciente, sinais clínicos, e exames complementares como radiografia ou endoscopia (Venter et al., 2005). A maioria dos corpos estranhos pode ser vistos em estudos radiográficos simples evidenciando o aumento da radiopacidade em casos de tecido ósseo (Thompson et al., 2012). Cabe ressaltar que a adição de contraste melhora o delineamento dos corpos radioluscentes. A ecografia e a endoscopia são também indicadas para o diagnóstico, podendo a endoscopia ser utilizada para a remoção e desobstrução do corpo estranho caso o mesmo não apresente superfície irregulares e margens afiadas potencialmente capaz de provocar lacerações na mucosa esofágica (Birchard \& Sherding, 2008; Sherding, 1994).

A remoção cirúrgica é amplamente utilizada devido a gama de possibilidades, e a escolha do procedimento e protocolo varia de acordo com a localização, tamanho, forma do corpo estranho e grau da obstrução, como é o caso da toracotomia. O esôfago torácico é de difícil acesso e requer técnicas meticulosas, pois esse órgão apresenta particularidades anatômicas e fisiológicas que requerem manipulação cuidadosa e delicada em abordagens cirúrgicas para que não haja prejuízo de cicatrização (Fossum, 2014). 
O objetivo do presente trabalho é relatar um caso de esofagotomia torácica para a remoção de um corpo estranho esofágico na porção torácica de um cão.

\section{Relato de caso}

Foi atendido um cão, fêmea, quatro anos, da raça Shih Tzu, pesando $4 \mathrm{~kg}$, com relato de ingestão de fragmento ósseo na noite anterior, desde então apresentava quadros de êmese pós-prandial, desconforto e dificuldade na ingestão de água. O animal também apresentava histórico de dor ao ser suspenso e transportado, porém não souberam especificar a região.

Ao exame clínico geral o animal apresentava hipertermia, taquipneia, taquicardia, desidratação e dor abdominal intensa. A partir da avaliação clínica o paciente foi primeiramente estabilizado com oxigenioterapia, venóclíse para fornecimento de fluidoterapia e medicações de suporte, como o uso de cloridrato de tramadol $4 \mathrm{mg} / \mathrm{kg}$, dipirona $25 \mathrm{mg} / \mathrm{kg}$, cloridrato de ranitidina $2 \mathrm{mg} / \mathrm{kg}$, ondansetrona 0,1 $\mathrm{mg} / \mathrm{kg}$, metronidazol $15 \mathrm{mg} / \mathrm{kg}$ e meloxicam $0,1 \mathrm{mg} / \mathrm{kg}$.

Depois de estabilizado o paciente foi realizado radiografia torácica nas projeções laterais direita, esquerda e ventro-dorsal da região torácica. Nas imagens a traqueia apresentava importante desvio ventral em relação ao esterno, desde a entrada do tórax até a base cardíaca, devido a uma estrutura óssea presente no esôfago torácico. Não foram visualizadas imagens compatíveis com estenose/colapso traqueal.

As três projeções realizadas no esôfago laterais e dorsal a silhueta cardíaca apresentaram uma estrutura radiopaca compatível com tecido ósseo (Figura 1) em formato geométrico semelhante a letra "L", pontiaguda (três pontas) e medindo $3,88 \mathrm{~cm} \mathrm{x} \mathrm{4,1} \mathrm{cm} \mathrm{(altura} \mathrm{x} \mathrm{comprimento)} \mathrm{(Figura} \mathrm{2),} \mathrm{sugerindo}$ obstrução esofágica. Nas demais estruturas e órgãos não foram visualizados alterações.

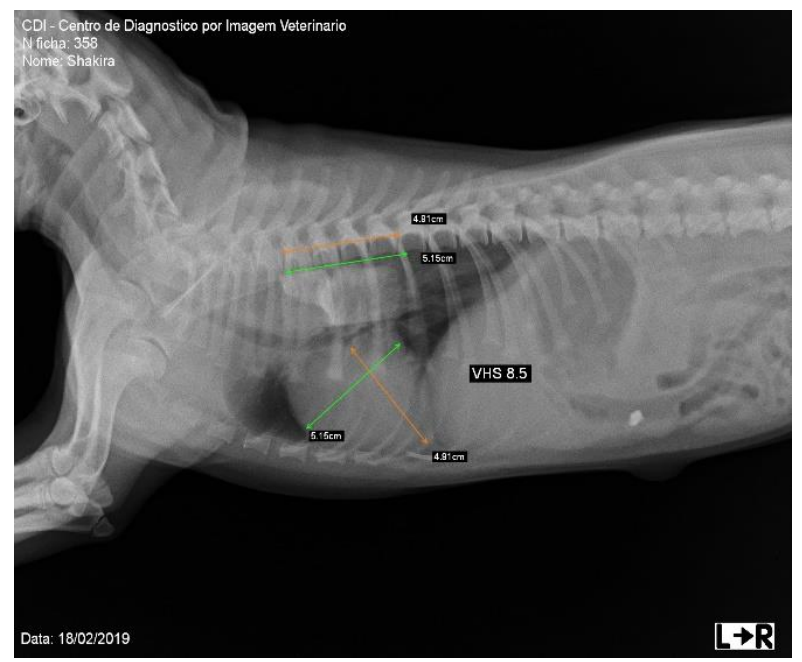

Figura 1. Radiografia em projeção lateral esquerda identificando estrutura radiopaca compatível com tecido ósseo.

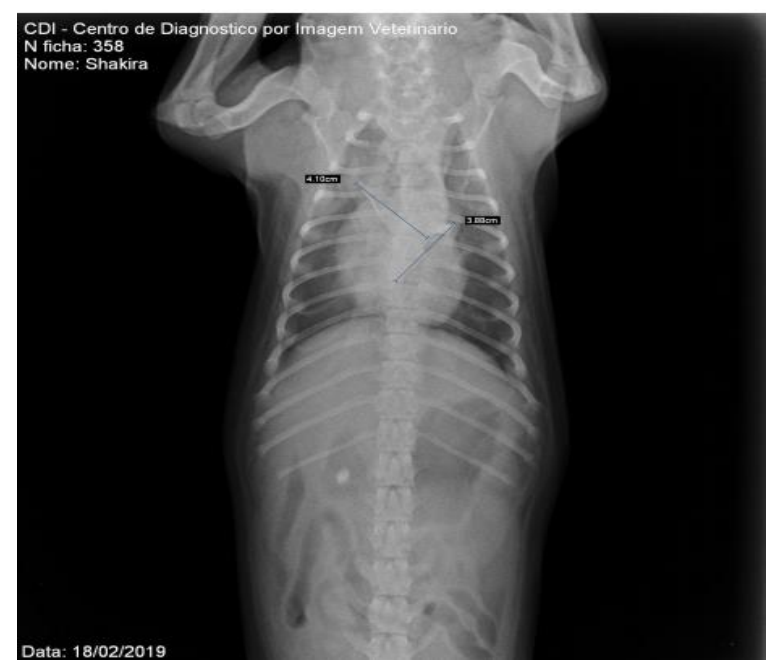

Figura 2. Radiografia em projeção ventro-dorsal identificando tecido ósseo em formato geométrico semelhante a letra "L", com três pontas e medindo $3,88 \mathrm{~cm} \mathrm{x} 4,1 \mathrm{~cm}$ (altura $\mathrm{x}$ comprimento).

Após a confirmação da obstrução esofágica, bem como região em que estava alocado, o tamanho e a forma do osso, foi indicada realização de exame hematológico para a realização do procedimento cirúrgico e encaminhamento do paciente para a cirurgia.

Foram realizados os preparativos anestésicos e cirúrgicos, após avaliação do paciente foi aplicada a medicação pré-anestésica (MPA) com acepromazina $0,03 \mathrm{mg} / \mathrm{kg}$ e metadona $0,2 \mathrm{mg} / \mathrm{kg}$, sendo o animal induzido com propofol $4 \mathrm{mg} / \mathrm{kg}$ e mantido com isofluorano $1,2 \%$, sendo mantido por ventilação mecânica durante todo o procedimento. Para o procedimento cirúrgico o animal foi preparado iniciando por uma ampla tricotomia do tórax lateral direito, posicionado em decúbito lateral esquerdo, realizado a antissepsia da região e abordagem cirúrgica pela lateral direita. 
A técnica cirúrgica foi realizada segundo o protocolo cirúrgico descrito em literatura, o qual se inicia com incisão no sétimo espaço intercostal (Figura 3), com a ressalva de que antes de perfurar e adentrar ao tórax deve-se assegurar que o animal esteja estabilizado com o anestesista. A seguir foi acessada a cavidade torácica e averiguado os órgãos, identificando-se o esôfago caudal e ventralmente à aorta. Também foram visualizados e protegidos os ramos ventral e dorsal do nervo vago na face lateral do esôfago, e exposto, por meio de uma transecção dos ligamentos pulmonares, foi feito a esofagotomia e cuidadosamente o corpo estranho foi removido do lúmen esofágico (Figura 4). O esôfago, os planos musculares e as costelas foram aproximados e suturados com a colocação prévia de suturas e padrões rotineiros na literatura e na clínica. Após a finalização do procedimento cirúrgico, foi fixado um dreno torácico, removido o ar residual da cavidade torácica, utilizando cateter e seringa, com o objetivo de manter a pressão intrapleural.

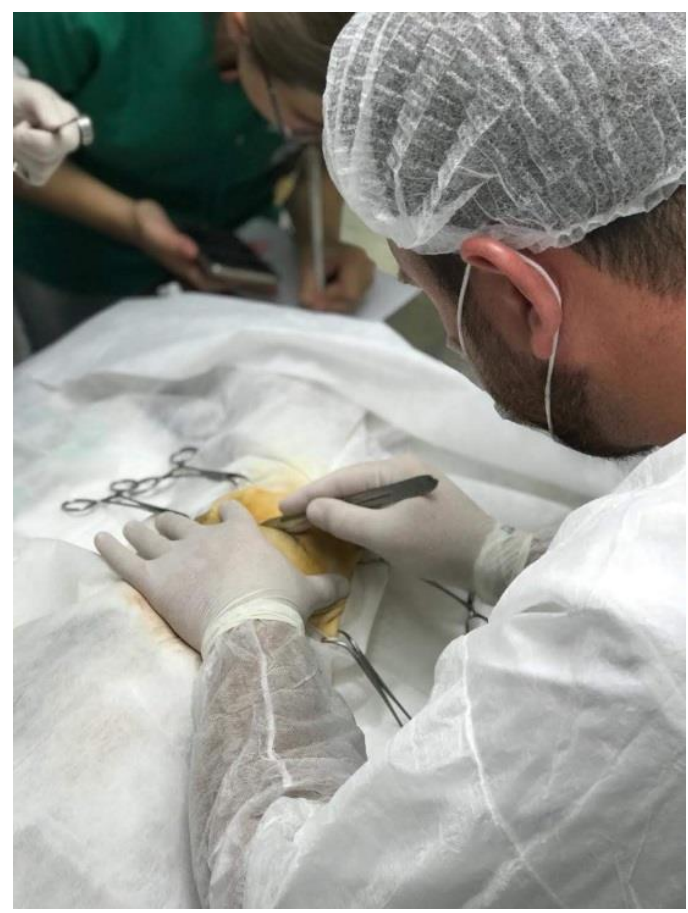

Figura 3. Incisão no sétimo espaço intercostal direito.

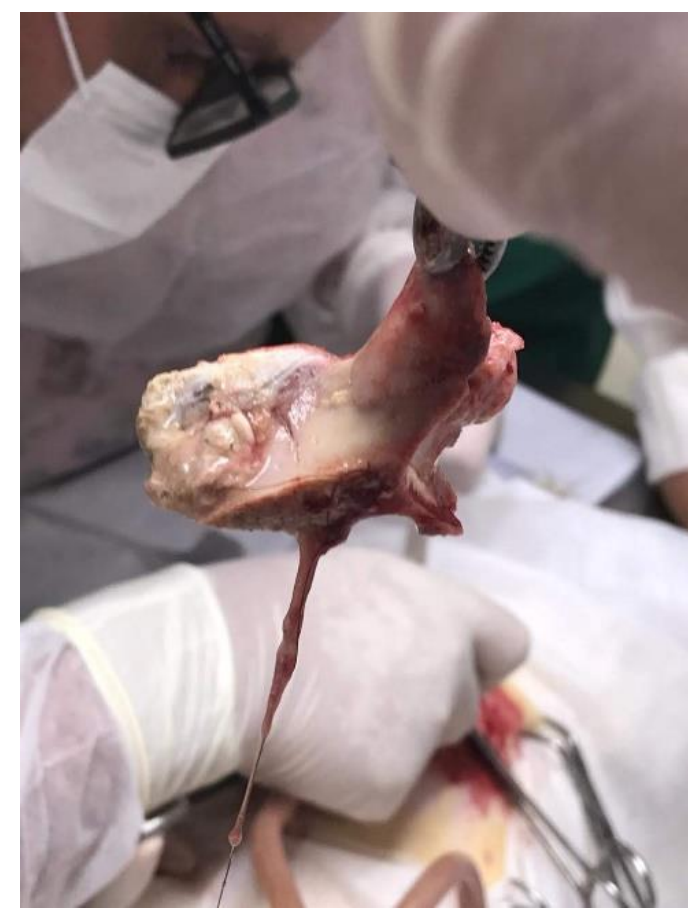

Figura 4. Remoção cuidadosa do corpo estranho no lúmen esofágico.

\section{Resultados e discussão}

No pós-cirúrgico, o animal se manteve estável e utilizando as mesmas medicações descritas anteriormente, respondendo bem ao procedimento e ao tratamento estabelecido, revelando um prognóstico favorável. Dessa forma, o paciente obteve alta médica após dois dias do procedimento cirúrgico, com as seguintes medicações: dipirona gotas $(25 \mathrm{mg} / \mathrm{kg})$, agemoxi $50 \mathrm{mg}(12,5 \mathrm{mg} / \mathrm{kg})$, e carproflan $25 \mathrm{mg}(2,2 \mathrm{mg} / \mathrm{kg})$. Foram recomendados cuidados como manter o animal em repouso durante este período, utilizar roupa cirúrgica ou colar elisabetano, fornecimento de uma dieta liquida/pastosa, água em frações pequenas, além de retornar, imediatamente, no caso de o paciente apresentar qualquer alteração.

Após o período de dez dias, o paciente retornou para avaliação e retirada da sutura, onde foi constatada uma ótima recuperação. Isso se deve, especialmente, à escolha do protocolo cirúrgico, pois, apesar de o corpo estranho estar localizado mais caudalmente no esôfago, o mesmo apresentava pontas e se encontrava próximo ao coração, optando-se pela realização da esofagotomia ao invés de uma gastrectomia, que é usualmente utilizado em situações similares. Dessa forma, evitou-se o risco de perfurações e acidentes durante o trans-cirúrgico, dando melhores condições de recuperação ao paciente.

As manobras anestésicas e as condutas pré e pós-operatórias condizem com os protocolos estabelecidos e presentes na literatura. Segundo Fossum (2014), a técnica cirúrgica abordada apresentou resultados positivos para o caso em relato por estar em concordância com a literatura. Cabe ressaltar que 
quanto maior a duração da obstrução, especialmente no caso de corpo estranho grande e aguçado, maior será a propensão do paciente para as complicações. Se há suspeita de corpo estranho instalado e o paciente está debilitado, deverão ser dados os passos diagnósticos necessários para que sejam delineadas todas as complicações secundárias (Slatter, 2007).

\section{Conclusão}

Os corpos estranhos esofágicos são frequentes na rotina do médico veterinário de pequenos animais, o diagnóstico deve-se iniciar pela anamnese e exame clínico geral, seguindo-se com exames complementares. Os tratamentos diferem e devem levar em consideração muitas variantes. Deve-se decidir o tratamento para cada caso e iniciá-lo assim que o animal estiver estabilizado. Sem complicações associadas o prognóstico é bom como ocorrido com o paciente relatado anteriormente, mas torna-se reservado quando há complicações decorrentes do corpo estranho, como perfuração esofágica.

\section{Referências bibliográficas}

Birchard, S. J. \& Sherding, R. G. (2008). Manual Saunders: clínica de pequenos animais (Vol. 3). São Paulo.

Fossum, T. W. (2014). Cirurgia de pequenos animais (4 ed. Vol. 1). São Paulo: Elsevier Brasil.

Juvet, F., Pinilla, M., Shiel, R. E. \& Mooney, C. T. (2010). Oesophageal foreign bodies in dogs: factors affecting success of endoscopic retrieval. Irish Veterinary Journal, 63(3):163-168.

Nelson, R. W. \& Couto, C. G. (2015). Medicina interna de pequenos animais. Amsterdan: Elsevier Editora.

Rodríguez, H., Passali, G. C., Gregori, D., Chinski, A., Tiscornia, C., Botto, H., . . Cuestas, G. (2012). Management of foreign bodies in the airway and oesophagus. International Journal of Pediatric Otorhinolaryngology, 76S84-S91.

Sherding, R. G. (1994). The cat: diseases and clinical management.

Slatter, D. H. (2007). Manual de cirurgia de pequenos animais. São Paulo: Manole.

Tans, T. R. (2005). Gastroenterologia de pequenos animais (Vol. 1). São Paulo: Editora Roca.

Tans, T. R. \& Rawlings, C. A. (2011). Small animal endoscopy. San Louis, USA: Elsevier Mosby.

Thompson, H. C., Cortes, Y., Gannon, K., Bailey, D. \& Freer, S. (2012). Esophageal foreign bodies in dogs: 34 cases (2004-2009). Journal of Veterinary Emergency and Critical Care, 22(2):253-261.

Venter, N. G., Jamel, N., Marques, R. G., Djahjah, F. \& Mendonça, L. d. S. (2005). Avaliação de métodos radiológicos na detecção de corpo estranho de madeira em modelo animal. Acta Cirurgica Brasileira, 20(1):19-26.

Recebido: 25 de junho, 2019.

Aprovado: 24 de julho, 2019.

Publicado: 13 de agosto, 2019.

Licenciamento: Este artigo é publicado na modalidade Acesso Aberto sob a licença Creative Commons Atribuição 4.0 (CC-BY 4.0), a qual permite uso irrestrito, distribuição, reprodução em qualquer meio, desde que o autor e a fonte sejam devidamente creditados. 